Supplement of Biogeosciences, 18, 4059-4072, 2021 https://doi.org/10.5194/bg-18-4059-2021-supplement (C) Author(s) 2021. CC BY 4.0 License.

(c) (1)

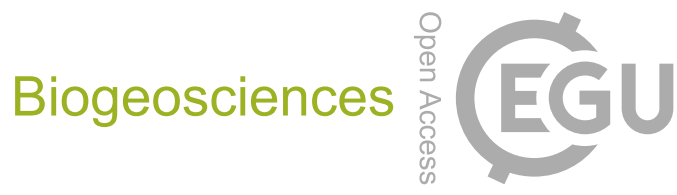

Supplement of

\title{
The motion of trees in the wind: a data synthesis
}

Toby D. Jackson et al.

Correspondence to: Toby D. Jackson (tobydjackson@gmail.com)

The copyright of individual parts of the supplement might differ from the article licence. 


\section{S1. Calculating the slope of the power spectrum}

45 In this paper we found that the slope of the power spectrum, $S_{\text {freq }}$, was a key feature of tree motion. Here we outline how it was calculated in detail and how sensitive the results are to the calculation method.

Each tree has two horizontal axes of motion and we applied a 10-minute high-pass Butterworth filter to remove offsets from each channel separately, and then calculated the resultant tree motion. We resampled each one-hour time-series to $4 \mathrm{~Hz}$ and calculated the power spectral density using Welch's method (pwelch, in Matlab). We then plot the log-transformed power spectral density (y-axis) against the log-transformed frequency (x-axis) and fit a linear model to the data across the specified frequency range to obtain the slope of the power spectrum, $S_{\text {freq. }}$. The fitting method and frequency range are somewhat arbitrary, and we test multiple options below. We noted that the high frequency range showed the most variation and the frequency ranges up to $2 \mathrm{~Hz}$ were more significant in the analyses, we therefore test this frequency range in more detail.
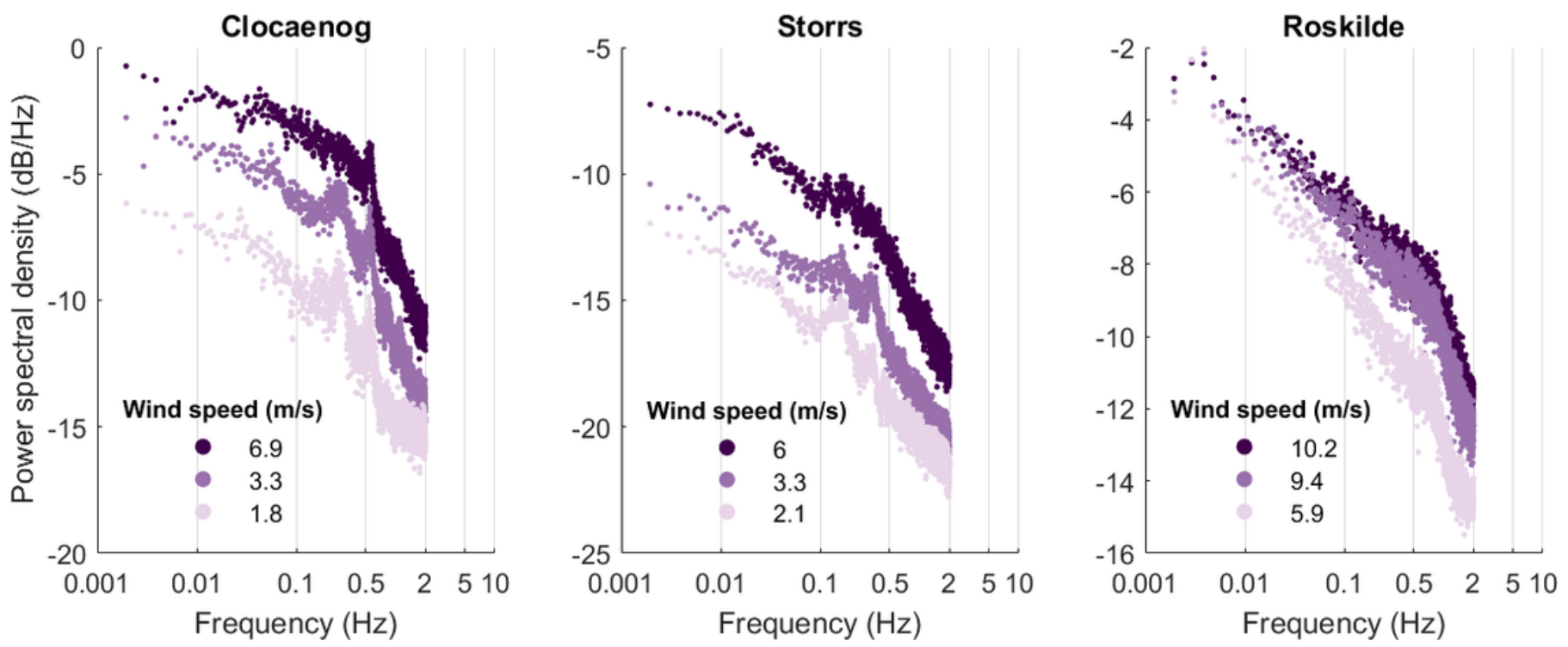

Figure S1 - Power spectra for example forest conifer (left), forest broadleaf (middle) and open-grown broadleaf (right) in high medium and low wind speeds (colours). 
60 We tested two methods to fit linear models to the power spectra: (1) using the output of pwelch directly and (2) logarithmically re-sampling the output to give evenly distributed log-transformed data. We found that this re-sampling altered the absolute values of the slope slightly, but did not alter the observed trends.
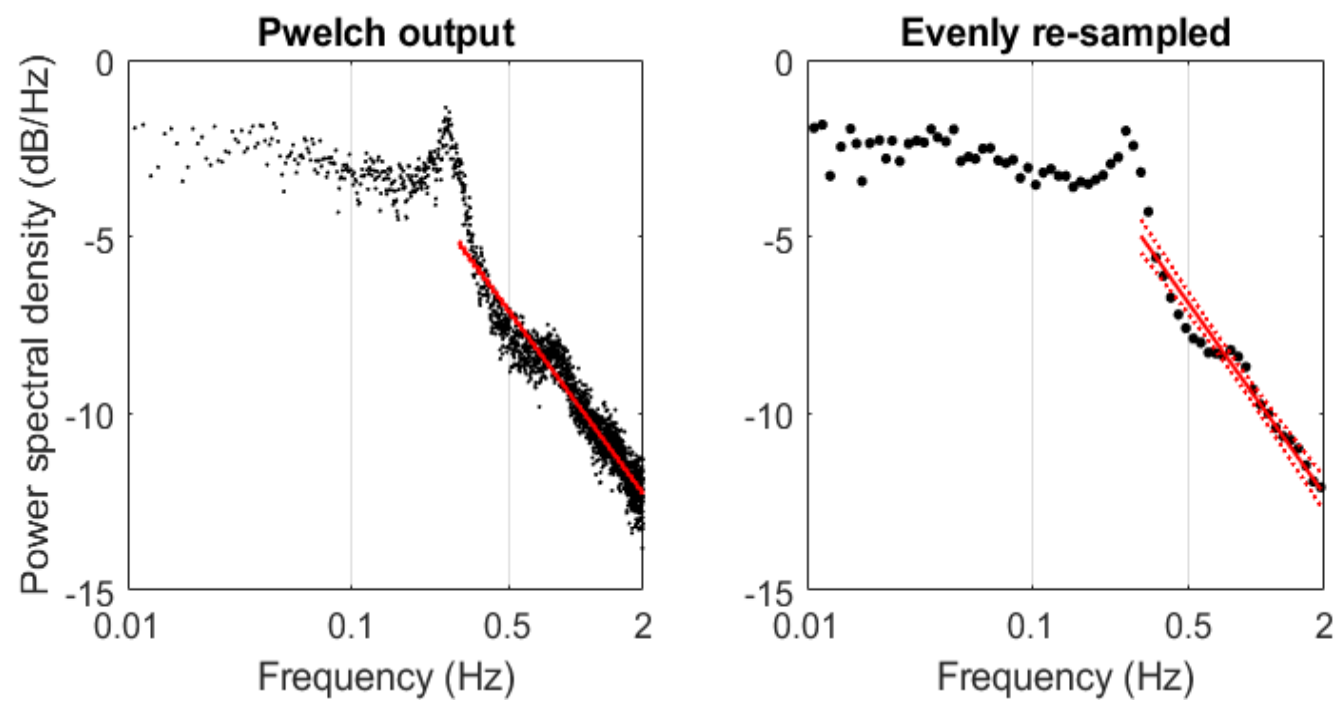

Figure S2 - Linear models of the power spectra to calculate slope using output of pwelch directly (left) and logarithmically resampled output (right). The frequency interval used in the above is $0.3-2 \mathrm{~Hz}$.

We tested three frequency intervals over which to fit the linear models, 0.05-2, 0.3-2 and 1-2 Hz. The first two intervals produced similar trends with slightly different absolute values. The shortest interval had a similar trend but was partially obscured by an increased level of noise.
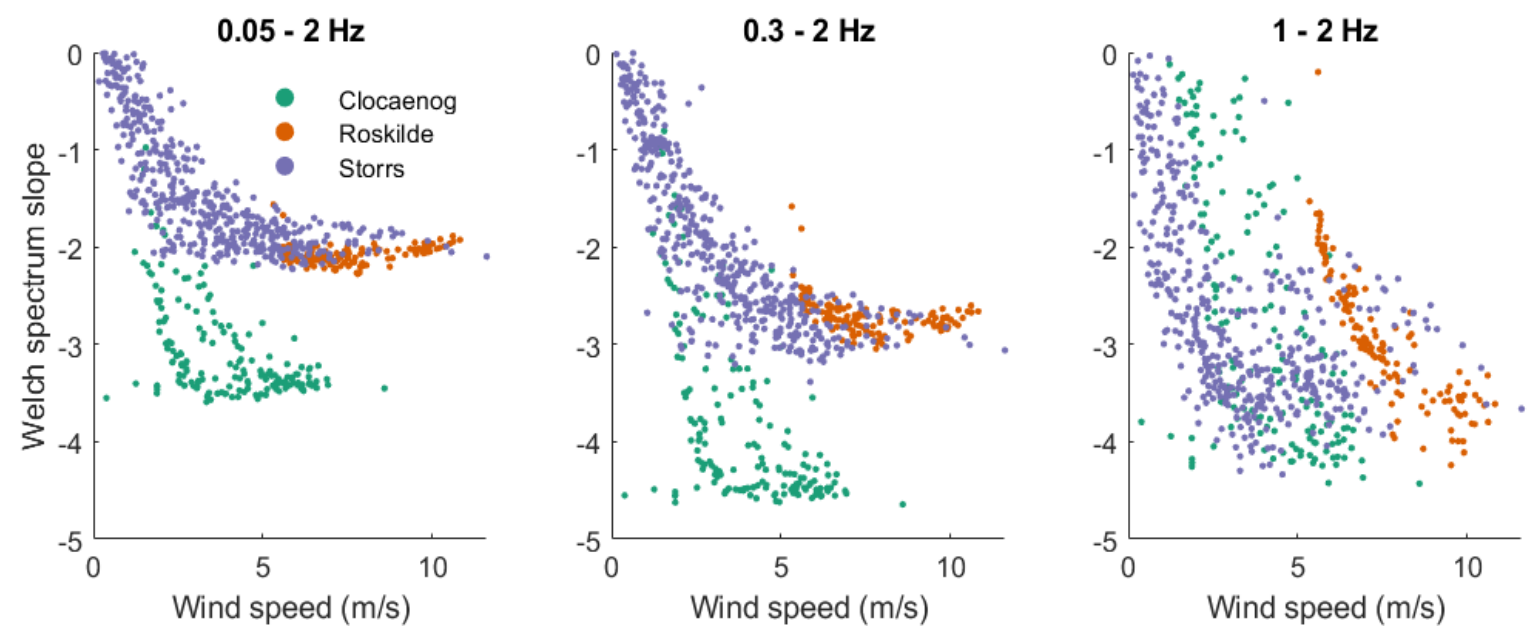
Figure S3 - Slope of the power spectrum against wind speed for three example trees (same as Figure S1) for three different frequency ranges.
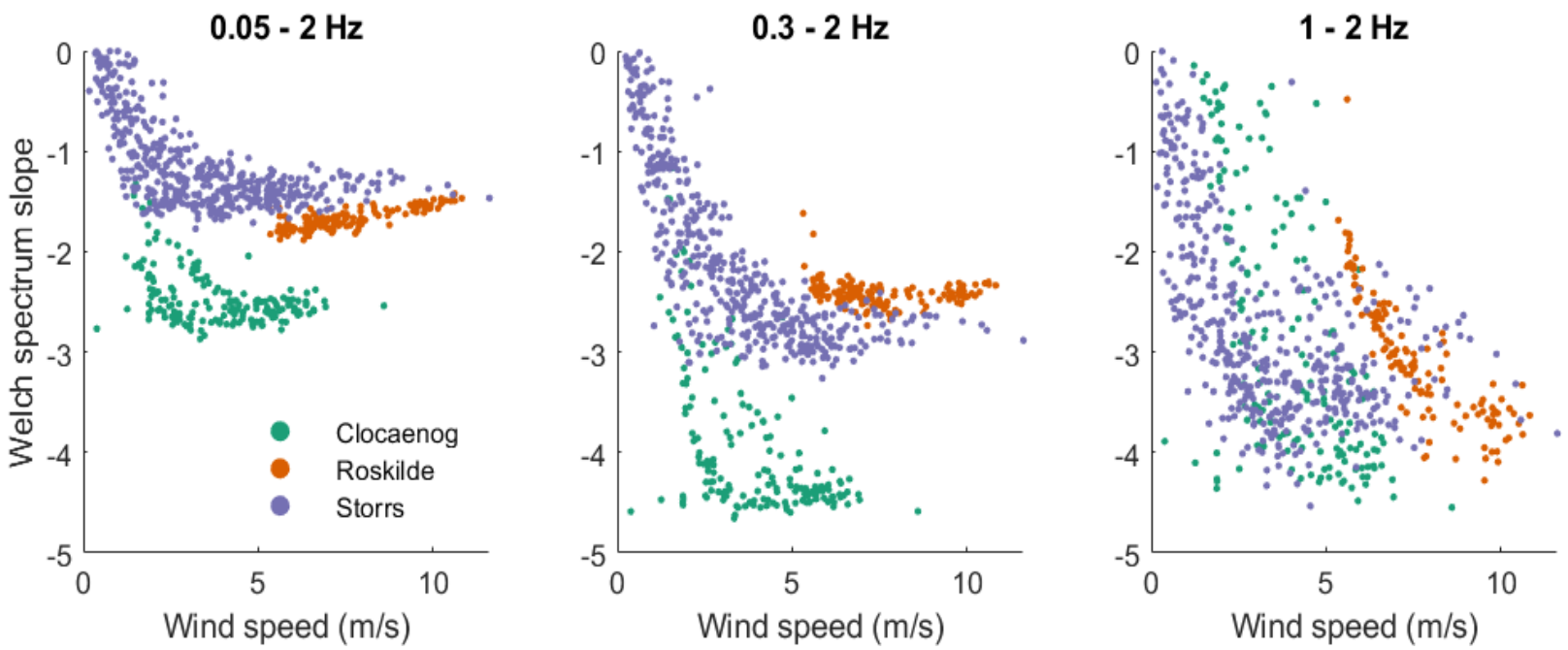

Figure S4 - Same as figure S3 but calculated using the logarithmically re-sampled power spectrum output.

Overall, we find that the trend described in Figure $4 \mathrm{~d}$ of the main text is robust to the different frequency ranges and fitting methods.

80 We also tested the effect of the Butterworth high-pass filter on the power spectra. The purpose of this filter is to remove offsets in the tree motion data. These offsets vary slowly so we chose a 10-minute high-pass filter. We found this had no significant effect on the power spectrum in the region of interest $(0.05-2 \mathrm{~Hz})$. 

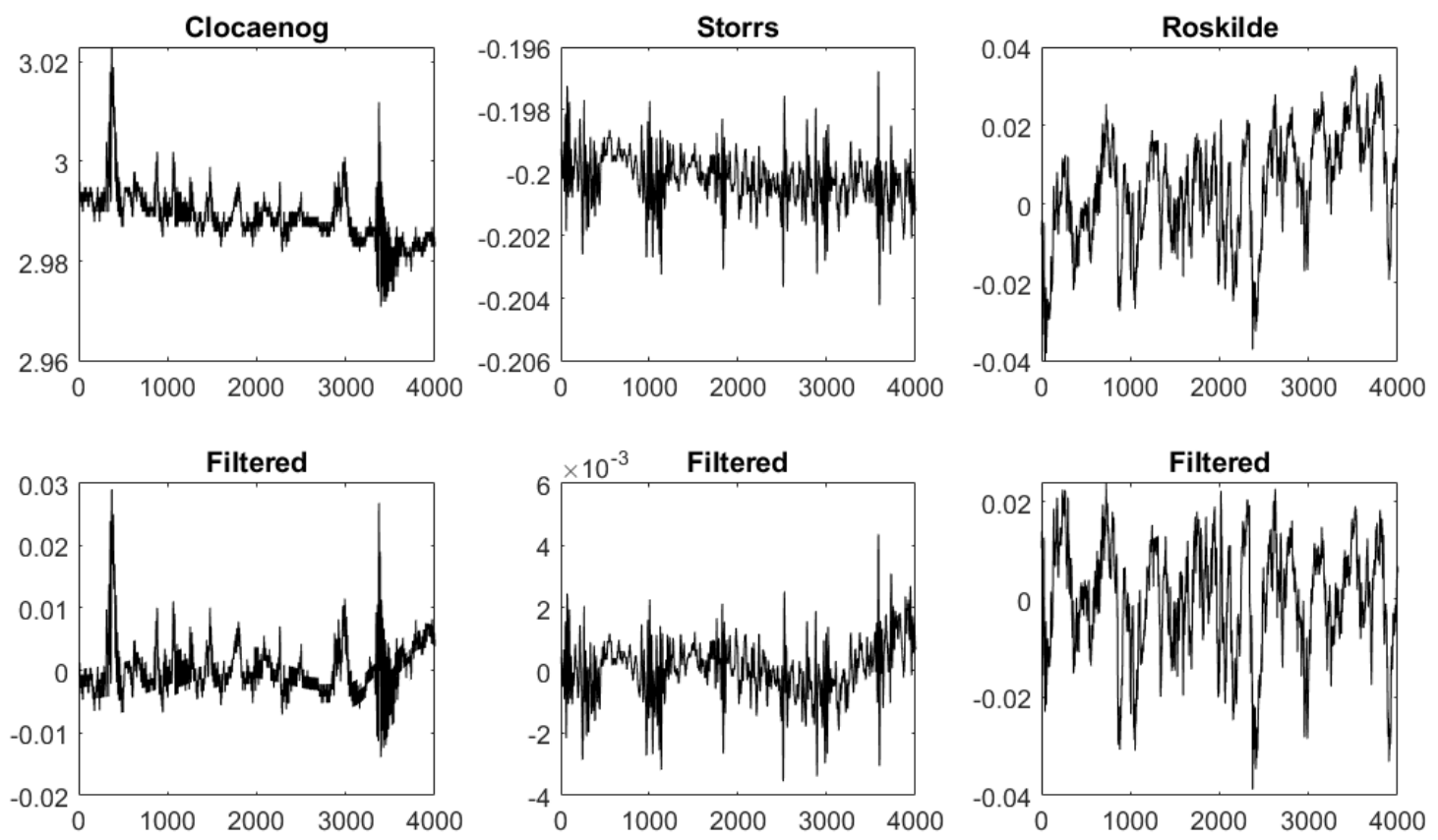

85 Figure S5 - Time-series for the same three trees as figures S1, 3 and 4 with and without the Butterworth high-pass filter.

\section{S2. Example power spectra at original resolution}

In the following we provide power spectra of tree motion (colours) and locally measured wind speed time-series (black) for the sites in which we have sufficient data. These are the forest broadleaf trees (figure S5) and forest conifers (figure S6). These power spectra were calculated at the original sampling frequency (all analysis in the main text was re-sampled to $4 \mathrm{~Hz}$ ) and we

95 therefore pre-multiplied the y-axes by the frequency to allow a direct comparison between sites. 

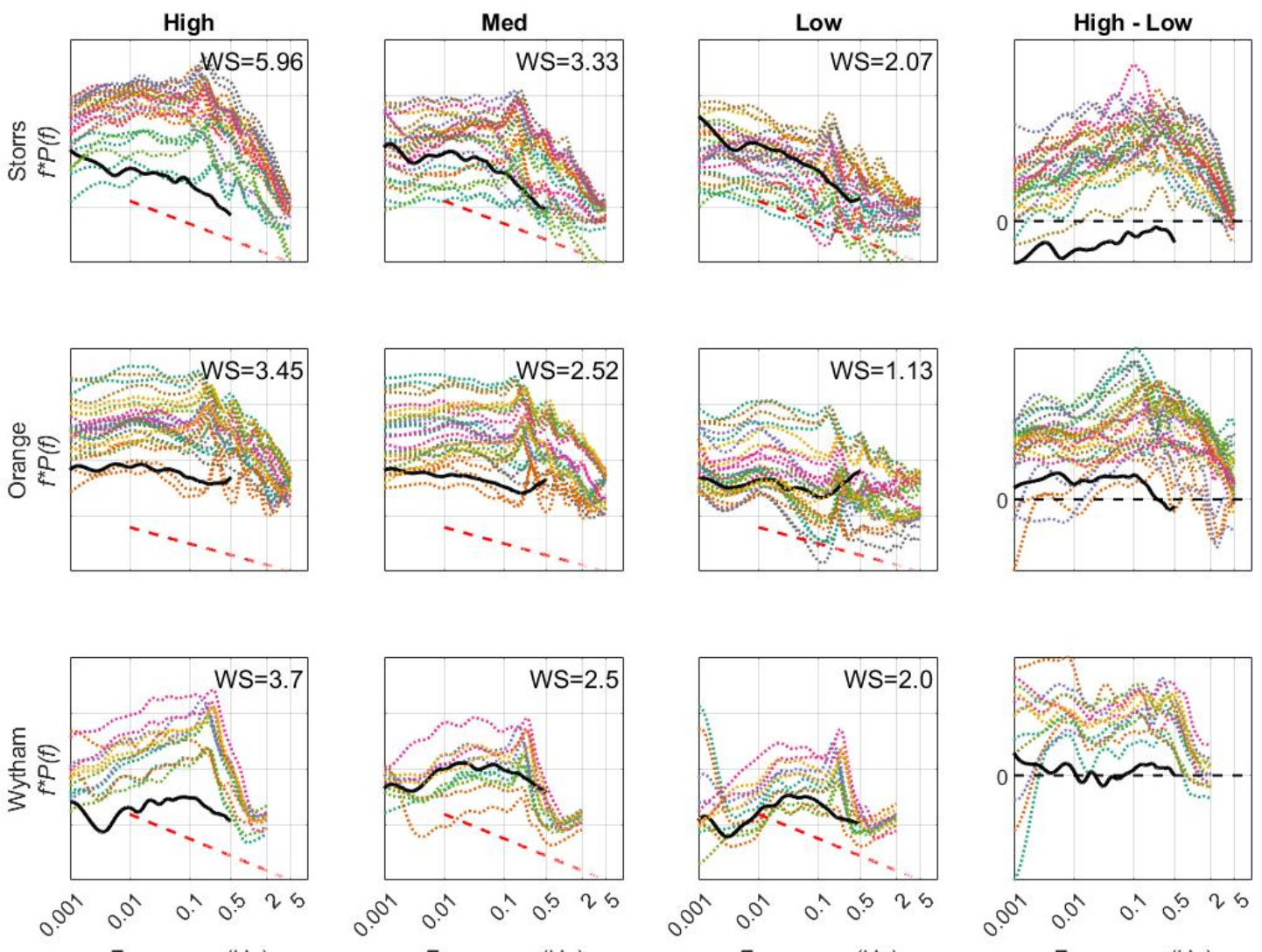

Frequency $(\mathrm{Hz})$
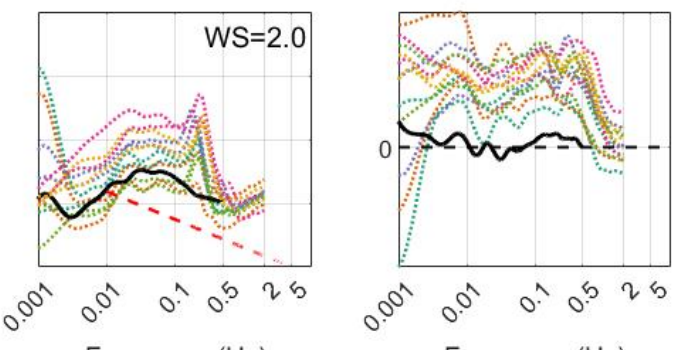

Figure S6 - power spectra for hourly samples of tree motion data (coloured lines) and wind speeds (black lines) at high, medium and low wind speed. Y-axis labels are the site names. The y-axis can be thought of as a measure of the relative energy content, in arbitrary units. The red dashed lines show the $-2 / 3$ slope as a reference point. The right-hand panels shows the difference between high and low wind speeds and the horizontal dashed line represents 0 change. Numbers in the top right-hand corners show the mean hourly wind speeds for the data sample. All of these sites are forest broadleaf trees. 

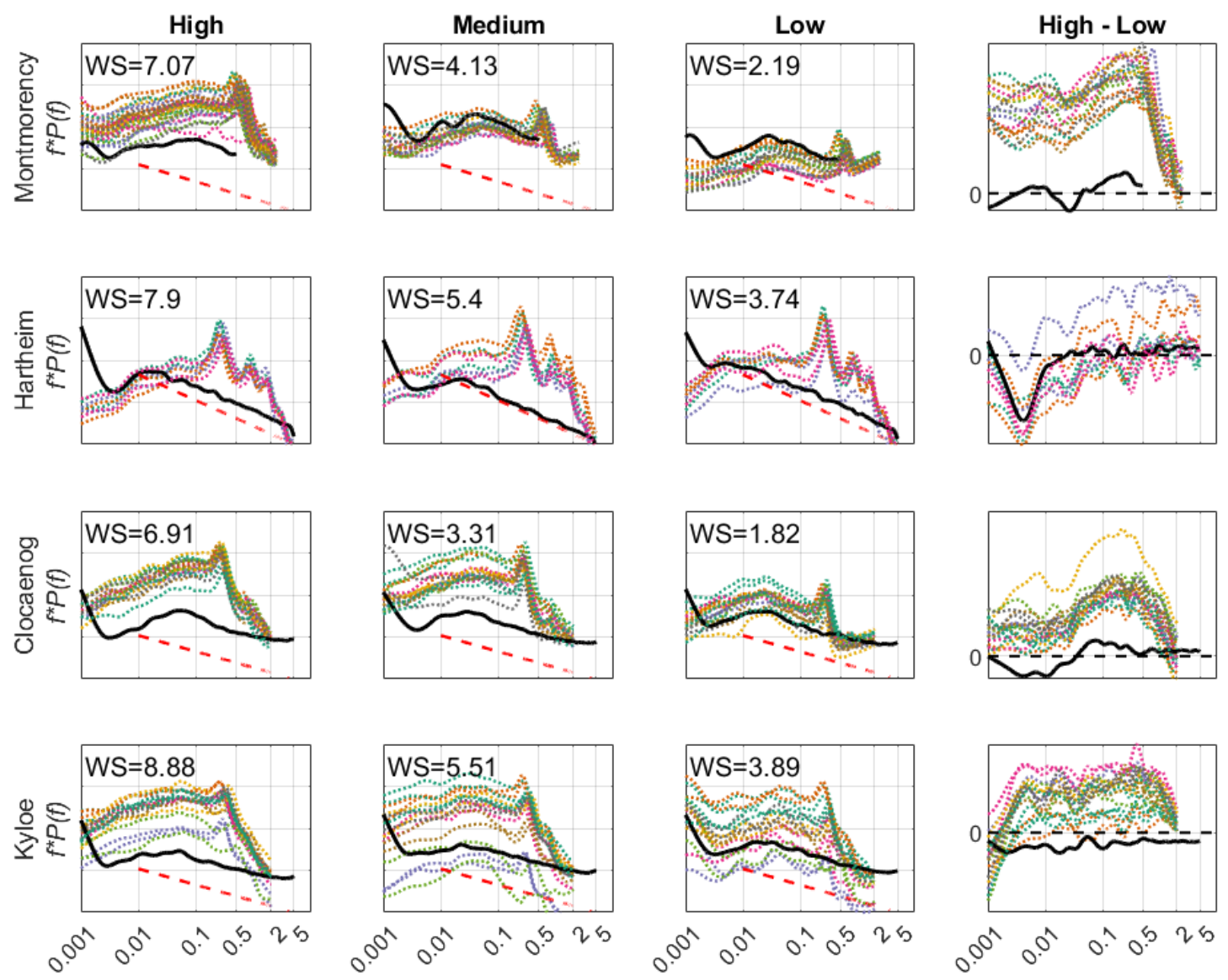

Frequency $(\mathrm{Hz})$

Frequency $(\mathrm{Hz})$

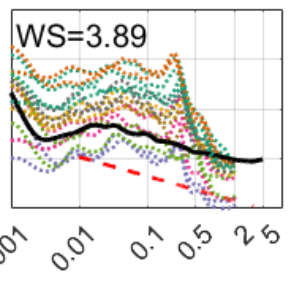

Frequency $(\mathrm{Hz})$

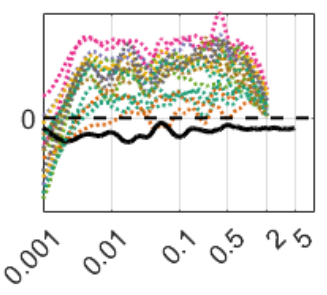

Frequency $(\mathrm{Hz})$

Figure S7 - Same as figure S6 but for sites in conifer forests. 


\section{S3. Correlation of all tree motion features}

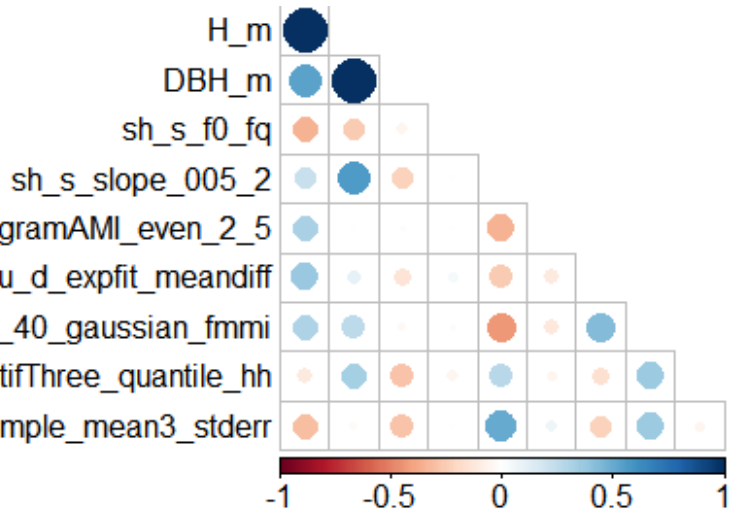

sh_s_CO_HistogramAMl_even_2_5 sh_s_CO_Embed2_Dist_tau_d_expfit_meandiff sh_S_IN_AutoMutuallnfoStats_40_gaussian_fmmi

sh_s_SB_MotifThree_quantile_hh sh_s_FC_LocalSimple_mean3_stderr sh_s SB Binarystats mean longstretch1 sh_s_SB_TransitionMatrix_3ac_sumdiagcov sh_s_PD_PeriodicityWang_th0_01 sh s CO Embed2 Dist tau d expfit meandiff sh_s_IN_AutolMutuallin foStats_ 40 - gaussian_fmmi sh_s_FC_LocalSimple_mean1_tauresrat sh_s_DN_Outlierinclude_p_001_mdrmd sh s DN Outlierlnclude n 001 mdrmd sh_s_SP_Summaries_welch_rect_area_5 1 sh_s_SB_BinaryStats_diff_longstretcho sh s SB MotifThree quantile hh sh_s_SC_FluctAnal_2_rsrangefit_50_1_logi_prop_r1 sh_s_SC_FluctĀnal_2_dfa_50_1_2_logi_prop_r1 sh_s_SP_Summaries_welch_rect_centroid sh_s_FC_LocalSimple_mean3_stderr

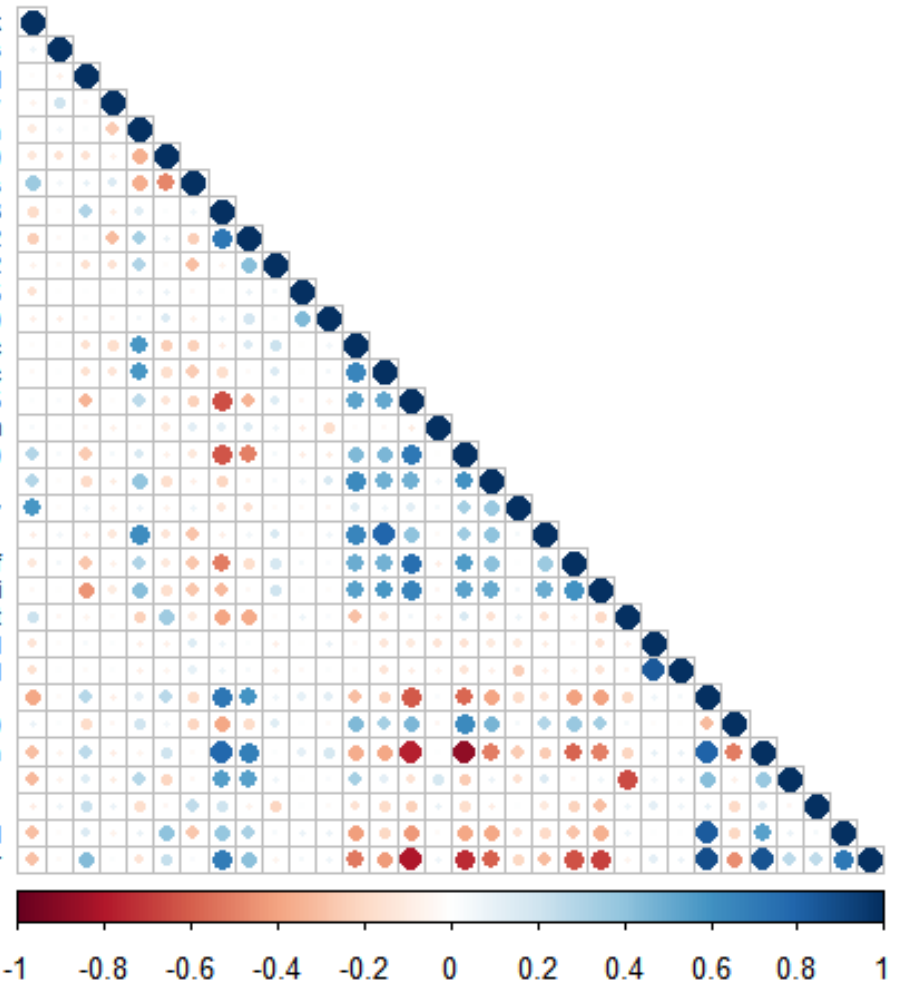

120 Figure S8 - Correlation plot for the tree motion features. Top panel shows tree height and diameter and only those features which are strongly correlated with them $\left(R^{2}>0.3\right)$. Bottom panels show all features used in this study. Colours represent strength of correlation as determined by the coefficient of determination $\left(\mathbf{R}^{2}\right)$ 
We considered a subset of trees $(\mathrm{N}=168,86$ forest broadleaves, 54 forest conifers and 28 open-grown broadleaves) for which height, $d b h$ and tree motion data were available. In order to test which features were closely related to tree size, while accounting for tree types, we predicted tree height and diameter from the tree motion features using a multiple linear regression including tree type in the model as a factor (Table 3). The best single predictor of $d b h$ was $S_{\text {freq }}$ while $f_{0}$ was the second best 130 predictor of tree height after a catch22 feature (CO_Embed2_Dist_tau_d_expfit_meandiff). The factor "tree type" was the $9^{\text {th }}$ most explanatory feature in the model of height and $6^{\text {th }}$ in the model of $d b h$. This demonstrates that tree size is more strongly related to tree motion features than it is to tree type. Therefore, the relationship between tree type and tree motion features is unlikely to be confounded by differences in tree size, and hence the results of our classification analyses are valid.

\begin{tabular}{|c|c|c|}
\hline Model for DBH & $\mathbf{R}^{2}$ & AIC \\
\hline Power spectral slope $\left(S_{\text {freq }}\right) 0.05-2 \mathrm{~Hz}$ & 0.308 & 174 \\
\hline IN_AutoMutualInfoStats_40_gaussian_fmmi & 0.356 & 163 \\
\hline CO_FirstMin_ac & 0.396 & 152 \\
\hline SB_MotifThree_quantile_hh & 0.426 & 144 \\
\hline FC_LocalSimple_mean3_stderr & 0.477 & 128 \\
\hline DN_Outlierlnclude_p_001_mdrmd & 0.494 & 123 \\
\hline Power spectral slope $\left(S_{\text {freq }}\right) 1-2 \mathrm{~Hz}$ & 0.509 & 119 \\
\hline CO_f1ecac & 0.530 & 111 \\
\hline Tree type & 0.546 & 108 \\
\hline
\end{tabular}

Table S1 - Summary statistics from the most parsimonious multiple linear models relating tree DBH to tree motion features. Each feature is added to the model sequentially in order of the largest decrease in AIC. A brief description of the catch22 features can be found in the next section (S5) and a more detailed description in the associated publication (Lubba et al 2019). 


\begin{tabular}{|c|c|c|}
\hline Model for height & $\mathbf{R}^{2}$ & AIC \\
\hline CO_Embed2_Dist_tau_d_expfit_meandiff & 0.127 & 1272 \\
\hline Fundamental frequency $\left(f_{0}\right)$ & 0.194 & 1259 \\
\hline SB_TransitionMatrix_3ac_sumdiagcov & 0.236 & 1250 \\
\hline Power spectral slope $\left(S_{\text {freq }}\right) 0.05-2 \mathrm{~Hz}$ & 0.286 & 1239 \\
\hline PD_PeriodicityWang_th0_01 & 0.318 & 1232 \\
\hline Number of wavelet peaks & 0.329 & 1230 \\
\hline Tree type & 0.353 & 1226 \\
\hline SP_Summaries_welch_rect_centroid & 0.364 & 1224 \\
\hline CO_HistogramAMI_even_2_5 & 0.385 & 1219 \\
\hline
\end{tabular}

150

Table S2 - Summary statistics from the most parsimonious multiple linear models relating tree height to tree motion features. Each feature is added to the model sequentially in order of the largest decrease in AIC. A brief description of the catch22 features can be found in the supplementary materials (S4) and a more detailed description in the associated publication (Lubba et al 2019).

\section{S5. Catch22 features table}




\begin{tabular}{|c|c|c|}
\hline & Name & Description \\
\hline $\mathrm{a}$ & DN_HistogramMode_5 & Mode of z-scored distribution (5-bin histogram) \\
\hline $\mathrm{b}$ & DN_HistogramMode_10 & Mode of z-scored distribution (10-bin histogram) \\
\hline $\mathrm{c}$ & SB_BinaryStats_mean_longstretch1 & Longest period of consecutive values above the mean \\
\hline $\mathrm{d}$ & DN_OutlierInclude_p_001_mdrmd & $\begin{array}{l}\text { Time intervals between successive extreme events above the } \\
\text { mean }\end{array}$ \\
\hline $\mathrm{e}$ & DN_OutlierInclude_n_001_mdrmd & $\begin{array}{l}\text { Time intervals between successive extreme events below the } \\
\text { mean }\end{array}$ \\
\hline f & CO_flecac & First 1/e crossing of autocorrelation function \\
\hline $\mathrm{g}$ & CO_FirstMin_ac & First minimum of autocorrelation function \\
\hline $\mathrm{h}$ & SP_Summaries_welch_rect_area_5_1 & $\begin{array}{l}\text { Total power in lowest fifth of frequencies in the Fourier power } \\
\text { spectrum }\end{array}$ \\
\hline $\mathrm{i}$ & SP_Summaries_welch_rect_centroid & Centroid of the Fourier power spectrum \\
\hline $\mathrm{j}$ & FC_LocalSimple_mean3_stderr & Mean error from a rolling 3-sample mean forecasting \\
\hline $\mathrm{k}$ & CO_trev_1_num & Time-reversibility statistic, $\mathrm{h}(\mathrm{xt}+1-\mathrm{xt}) 3 \mathrm{it}$ \\
\hline 1 & CO_HistogramAMI_even_2_5 & Automutual information, $\mathrm{m}=2,=5$ \\
\hline $\mathrm{m}$ & IN_AutoMutualInfoStats_40_gaussian_fmmi & First minimum of the automutual information function \\
\hline $\mathrm{n}$ & MD_hrv_classic_pnn40 & Proportion of successive differences exceeding 0.04 \\
\hline o & SB_BinaryStats_diff_longstretch0 & Longest period of successive incremental decreases \\
\hline $\mathrm{p}$ & SB_MotifThree_quantile_hh & $\begin{array}{l}\text { Shannon entropy of two successive letters in equiprobable 3- } \\
\text { letter symbolization }\end{array}$ \\
\hline q & FC_LocalSimple_mean1_tauresrat & Change in correlation length after iterative differencing \\
\hline $\mathrm{r}$ & CO_Embed2_Dist_tau_d_expfit_meandiff & Exponential fit to successive distances in 2-d embedding space \\
\hline $\mathrm{s}$ & SC_FluctAnal_2_dfa_50_1_2_logi_prop_r1 & $\begin{array}{l}\text { Proportion of slower timescale fluctuations that scale with DFA } \\
\text { (50\% sampling) }\end{array}$ \\
\hline $\mathrm{t}$ & SC_FluctAnal_2_rsrangefit_50_1_logi_prop_r1 & $\begin{array}{l}\text { Proportion of slower timescale fluctuations that scale with } \\
\text { linearly rescaled range fits }\end{array}$ \\
\hline $\mathrm{u}$ & SB_TransitionMatrix_3ac_sumdiagcov & $\begin{array}{l}\text { Trace of covariance of transition matrix between symbols in 3- } \\
\text { letter alphabet }\end{array}$ \\
\hline $\mathrm{v}$ & PD_PeriodicityWang_th0_01 & $\begin{array}{l}\text { Periodicity measure of Wang, X., Wirth, A., Wang, L.: } \\
\text { Structure-based statistical features and multivariate } \\
\text { time series clustering. Proceedings - IEEE International } \\
\text { Conference on Data Mining, } \\
\text { ICDM pp. 351-360 (2007). }\end{array}$ \\
\hline
\end{tabular}

